Fixed Point Theory, 21(2020), No. 1, 125-132

DOI: $10.24193 /$ fpt-ro.2020.1.09

http://www.math.ubbcluj.ro/ nodeacj/sfptcj.html

\title{
A COMMON MAXIMAL ELEMENT OF CONDENSING MAPPINGS
}

\author{
LIANG-JU CHU* AND CHIEN-HAO HUANG** \\ *Department of Mathematics, NTNU, ROC \\ E-mail: chulj@math.ntnu.edu.tw \\ Corresponding author \\ ** Department of Mathematics, NTNU, ROC \\ E-mail: qqnick0719@yahoo.com.tw
}

\begin{abstract}
In this paper, we establish a general existence theorem of maximal elements of condensing mappings in the product $X:=\prod_{\alpha \in I} X_{\alpha}$ of noncompact l.c.-spaces. As an application, we prove that a family of $\mathcal{L}_{\pi_{\alpha}}$-majorized $Q_{\alpha}$-condensing mappings $T_{\alpha}: X \longrightarrow 2^{X_{\alpha}}$ admit a common maximal element under the mild condition that each $\left\{x \mid T_{\alpha}(x) \neq \emptyset\right\}$ is compactly open.

Key Words and Phrases: l.c.-space, $Q_{\alpha}$-condensing mapping, maximal element, $\mathcal{L}_{\theta}$-majorized. 2010 Mathematics Subject Classification: 47H04, 52A99, 54H25.
\end{abstract}

\section{REFERENCES}

[1] C. Bardaro, R. Ceppitelli, Some further generalizations of Knaster-Kuratowski-Mazurkiewicz theorem and minimax inequalities, J. Math. Anal. Appl., 132(1988), 484-490.

[2] X.P. Ding, W.K. Kim, K.K. Tan, Equilibria of non-compact generalized games with $\mathcal{L}^{*}$ majorized preference correspondences, J. Math. Anal. Appl., 164(1992), 508-517.

[3] Y. M. Du, Fixed points, maximal elements and equilibria of generalized games in abstract convex spaces, Abstract Applied Anal., 2012, Article ID 842515, 17 pages.

[4] W. Guo, Y.J. Cho, A new maximal element theorem in H-space with applications, Nonlinear Anal., 68(2008), 2194-2203.

[5] C.D. Horvath, Contractibility and generalized convexity, J. Math. Anal. Appl., 156(1991), 341357.

[6] J.L. Kelley, General Topology, Springer-Verlag Press, 1975.

[7] W.K. Kim, A maximal element of condensing multimaps, J. Chung. Math. Soc., 6(1993), 59-64.

[8] L.J. Lin, Q.H. Ansari, Collective fixed points and maximal elements with applications to abstract economies, J. Math. Anal. Appl., 296(2004), 455-472.

[9] G. Mehta, Maximal elements of condensing preference maps, Appl. Math. Lett., 3(2)(1990), 69-71.

[10] E. Tarafdar, A fixed point theorem in H-spaces and related results, Bull. Austral. Math. Soc., 42(1990), 133-140.

[11] E. Tarafdar, Fixed point theorems in H-spaces and equilibrium points of abstract economies, J. Austral. Math. Soc., Series A, 53(1992), 252-260.

This work is partially supported by the Ministry of Science and Technology of ROC.. 
[12] X. Wu, Existence theorem for maximal elements in $H$-spaces with applications on the minimax inequalities and equilibrium of games, J. Appl. Anal., 6(2000), 283-293.

[13] X. Wu, Z.F. Shen, Equilibrium of abstract economy and generalized quasi-variational inequality in H-spaces, Topol. Appl., 153(2005), 123-132.

[14] Y.L. Wu, C.H. Huang, L.J. Chu, An extension of Mehta Theorem with applications, J. Math. Anal. Appl., 391(2012), 489-495.

Received: October 20, 2016; Accepted: November 28, 2016, 